\title{
Socioeconomic status and cardiovascular risk SCORE
}

\author{
Ewa Podolecka ${ }^{1}$, Agnieszka Doryńska², Paweł Nadrowski ${ }^{1}$, Michał Skrzypek ${ }^{3}$, \\ Magdalena Kwaśniewska ${ }^{4}$, Wojciech Drygas ${ }^{4,5}$, Andrzej Pająk², Krystyna Kozakiewicz ${ }^{1}$ \\ $13^{\text {rd }}$ Department of Cardiology, Upper Silesian Centre of Cardiology, Medical University of Silesia, Katowice, Poland \\ ${ }^{2}$ Department of Epidemiology and Population Studies, Institute of Public Health, Jagiellonian University Medical College, Krakow, Poland \\ ${ }^{3}$ School of Public Health in Bytom, Department of Biostatistics, Medical University of Silesia, Katowice, Poland \\ ${ }^{4}$ Department of Preventive and Social Medicine, Medical University of Lodz, Lodz, Poland \\ ${ }^{5}$ Department of Epidemiology, Cardiovascular Disease Prevention and Health Promotion, Institute of Cardiology, Warsaw, Poland
}

\begin{abstract}
A b stract
Background: Cardiovascular diseases (CVD) are one of the most frequent causes of morbidity and death both in men and women. The influence of the following factors on the occurrence and progression of atherosclerosis is well known: hypertension, hypercholesterolaemia, tobacco smoking, obesity, diabetes, age, and sex. As well as the typical risk factors of CVD, there is also a significant association between the incidence of those diseases and socioeconomic status (SES).

Aim: The aim of this study was to establish the correlation between SES status and CVD risk assessed according to the SCORE algorithm.

Methods: The study encompassed 516 participants (207 men and 309 women) aged 40-74 years, who had never been diagnosed with any CVD. The SES was calculated by multiplying the patient's education and net monthly income. The correlation between the SES and SCORE was established using linear and logistic regression analysis.

Results: After considering the influence of age, an inverse correlation between the SCORE risk value and the SES index was established, both in the entire group $(p=0.006)$ and in the men's group $(p=0.007)$. In the analysis of individual age subgroups, this correlation was demonstrated in the following groups: 55-59-year-olds $(p=0.011), 60-64$-year-olds $(p=0.014)$, and $65-69-$ year olds $(p=0.034)$. A similar relationship was established in men aged $65-69$ years $(p=0.038)$ and women aged $40-44$ years $(p=0.003)$. The logistic regression analysis demonstrated that, after considering the influence of age, the odds of the SCORE risk value being $\geq 10 \%$ were becoming smaller along with the increase in the SES index value in the entire group $(p=0.048)$ and in the men's group $(p=0.011)$. The odds ratio $(O R)$ for the SCORE risk value being $\geq 10 \%$ depending on the SES index value was OR $=0.978$ (95\% confidence interval $[\mathrm{Cl}]$ 0.956-0.999) in the entire group and OR $=0.964(95 \%$ $\mathrm{CI}$ 0.938-0.992) in men. Furthermore, we also established that the risk of SCORE $\geq 5 \%$ decreased with the increase in the SES index value in the women's group $(\mathrm{OR}=0.970 ; 95 \% \mathrm{Cl} 0.941-0.999 ; \mathrm{p}=0.042)$

Conclusions: 1. We demonstrated a statistically significant correlation between the SES and the CVD risk assessed according to the SCORE algorithm. 2. The value of the CVD risk according to SCORE was inversely correlated with SES status.
\end{abstract}

Key words: cardiovascular risk, SCORE algorithm, socioeconomic status

Kardiol Pol 2018; 76, 3: 560-565

\section{INTRODUCTION}

Despite the advances in diagnostics and methods of treatment, cardiovascular diseases (CVD) are still one of the most frequent causes of morbidity and death both in men and women. As yet, a number of risk factors associated with the development of CVD have been established. The influence of the following factors on the occurrence and progression of atherosclerosis well known is: hypertension, hypercholesterolaemia, tobacco smoking, obesity, diabetes, age, and sex [1, 2]. In recent years, increasing attention has been focused on socioeconomic

\section{Address for correspondence:}

Ewa Podolecka, MD, PhD, $3^{\text {rd }}$ Department of Cardiology, Upper Silesian Centre of Cardiology, Medical University of Silesia, ul. Ziołowa 45/47, 40-635 Katowice, Poland, e-mail: epodolecka@interia.pl 
status (SES) as a risk factor of CVD. Many epidemiological studies have confirmed the correlation between the low SES and increased incidence of CVD [3-5]. This may be associated with a greater prevalence of such risk factors as tobacco smoking, hypertension, hypercholesterolaemia, diabetes, and obesity in socioeconomic groups of lower status [6, 7]. The aim of the study was to establish the correlation between the SES and the CVD risk assessed according to the Systematic Coronary Risk Evaluation (SCORE) algorithm in the population inhabiting the south-western part of Poland.

\section{METHODS}

The research is a part of the WOBASZ II project, which was conducted in Poland in the years 2013-2014 as a means of primordial prevention of CVD. A detailed description, methodology, and objectives of the WOBASZ II study are presented in a publication by Drygas et al. [8]. The analysis encompassed data collected from a random group of inhabitants of the Silesian, Lower Silesian, and Opolskie voivodeships. In each voivodeship, six gminas were randomly chosen for the study: two small (up to 8000 inhabitants), two medium (8000-40,000 inhabitants), and two large ones (40,000 inhabitants and more). Next, 70 men and 70 women aged 20 years and above were randomly selected from each gmina. The random selection was done among Polish citizens based on the data available in the department of National Registers of the Ministry of the Interior and Administration. The WOBASZ II study was approved by the Bioethics Commission of the Institute of Cardiology in Warsaw.

All participants filled in a questionnaire, providing socio-epidemiological data such as: age, sex, education, monthly net income per household member, and addictions (including tobacco smoking). Moreover, all participants were subject to history and physical examination as well as laboratory testing, which encompassed the estimation of glucose, total cholesterol, high-density lipoprotein (HDL) cholesterol, triglycerides, and low-density lipoprotein (LDL) cholesterol in serum (with overnight fasting). Each participant was assessed in terms of their SES, which was defined according to the method used in the ATTICA study, namely the product of education and net monthly income [9]. Individual categories of variables were provided with numerical values - education: incomplete primary $=1$, primary $=2$, basic vocational after primary school $=3$, middle school $=4$, basic vocational after middle school $=5$, high school/technical high school (secondary) $=6$, post-secondary $=7$, bachelor's degree (incomplete higher) $=8$, higher $=9$, and income: $\leq 500$ PLN $=1,501-1000$ PLN $=2,1001-$ $-1500 \mathrm{PLN}=3,1501-2000 \mathrm{PLN}=4,2001-2500 \mathrm{PLN}=5$, 2501-3000 PLN $=6$ and $>3001 \mathrm{PLN}=7$. The SES value was calculated by multiplying the patient's education and net monthly income. The SES groups were determined in the following manner: low SES - value $<8$, middle SES — value 8-18, high SES — value $>18$.
The random group comprised 884 participants. From the above-mentioned group, we selected 516 participants (207 men and 309 women) aged 40-74 years, who had never been diagnosed with any CVD. Based on the SCORE algorithm for the population of Poland, the CVD risk was assessed for each participant [10, 11]. The SCORE algorithm allows us to assess the risk of death from cardiovascular causes within 10 years based on the information regarding age, sex, systolic blood pressure, total cholesterol level, and tobacco smoking. Risk assessment through the SCORE algorithm and tables is designed to be used in primordial prevention in patients aged over 40 years, who have no cardiovascular incidents in their medical history. The high-risk group consists of patients without clinical symptoms of CVD, who received a risk value $\geq 5 \%$ based on the SCORE algorithm [10, 11].

\section{Statistical analysis}

To describe continuous variables we used the median (interquartile range), while in the case of categorical variables the occurrence frequency of individual categories was represented as a percentage (\%). We calculated the SES and SCORE indexes adjusted by age (weighted by age). We conducted linear regression analysis to assess the correlation between the SCORE risk value expressed as a continuous variable and the SES index, separately for the individual age and sex groups. We also conducted logistic regression analysis, which expressed the SCORE risk value as a categorical variable (an increased SCORE value) in the correlation analysis with the SES index, separately for each sex group. The significance level was defined as $p<0.05$. The analyses were conducted using a statistical software package STATA version 12.1 (StataCorp LP, Texas, USA).

\section{RESULTS}

The descriptive characteristics of the analysed variables in the entire group under study as well as in the sex and age groups are presented in Tables 1-3. The median of the SES index value weighted by age was 12 , both in the entire group and in the men's and women's groups. We observed a downward trend in the SES median value along with the increase in participants' age. $28.5 \%$ of participants were classified into the low SES category (27.0\% of men and $28.8 \%$ of women). $29.8 \%$ of participants were classified into the high SES category (33.6\% of men and $30.6 \%$ of women). The median SCORE risk value was 1.66 in the entire group (2.87 in men and 0.95 in women) and, in contrast to the SES value, it demonstrated an upward tendency with the increase in age (Tables 1-3).

The results of linear regression analysis demonstrated that, after considering the influence of age, a correlation between the SCORE risk value and the SES index was observed in the entire group $(p=0.006)$ and in the men's group $(p=0.007)$. No correlation was observed in the women's group. The SCORE risk value decreased in the entire group by 0.047 of a unit along with the increase in the SES index by 

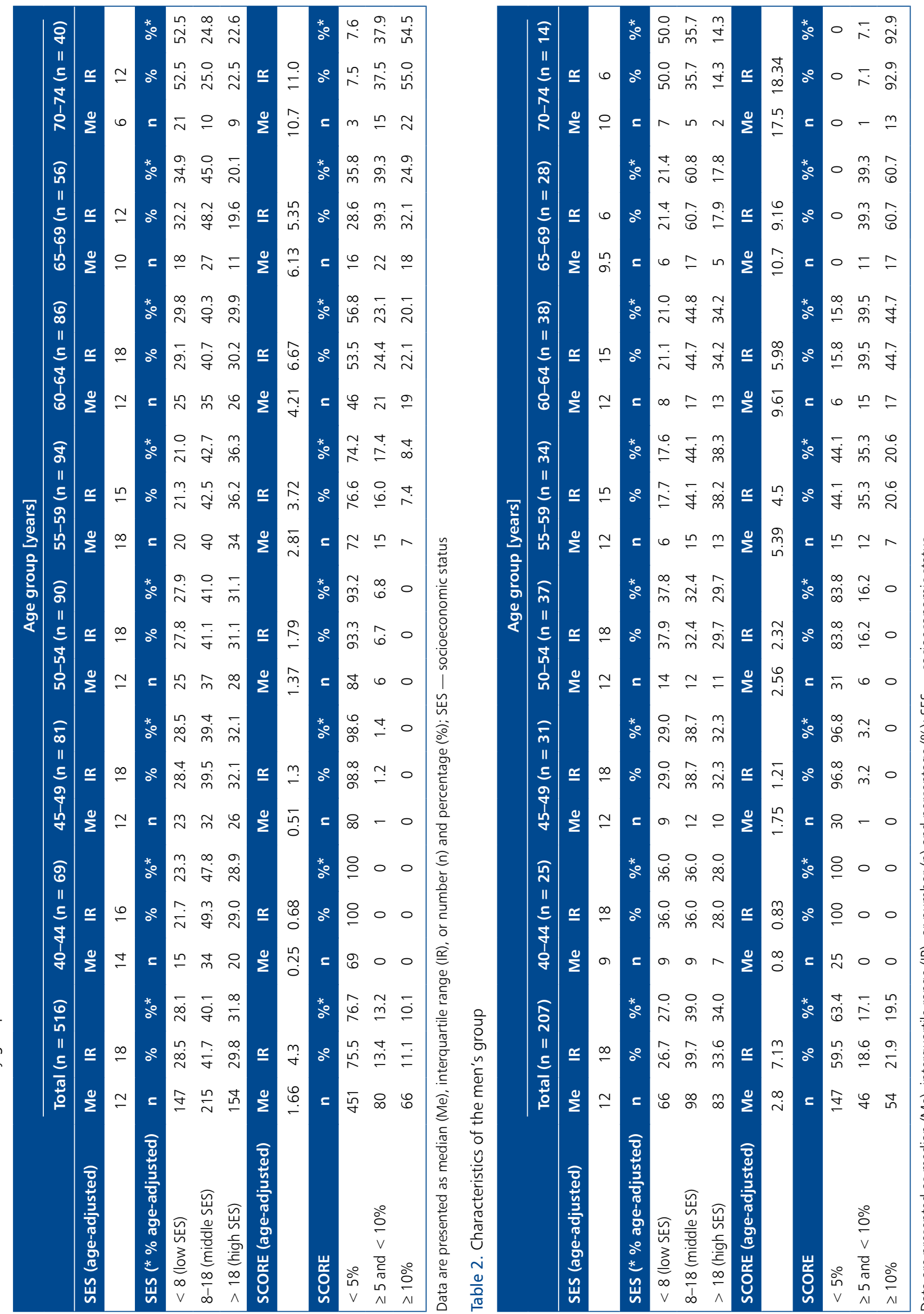

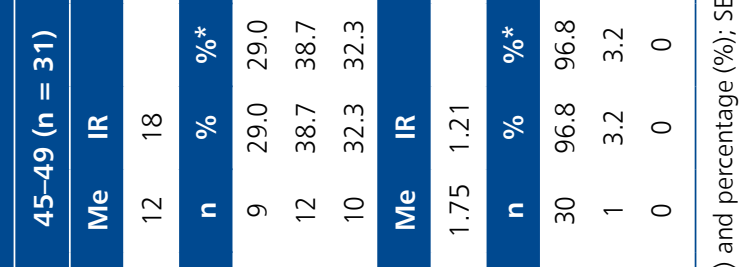
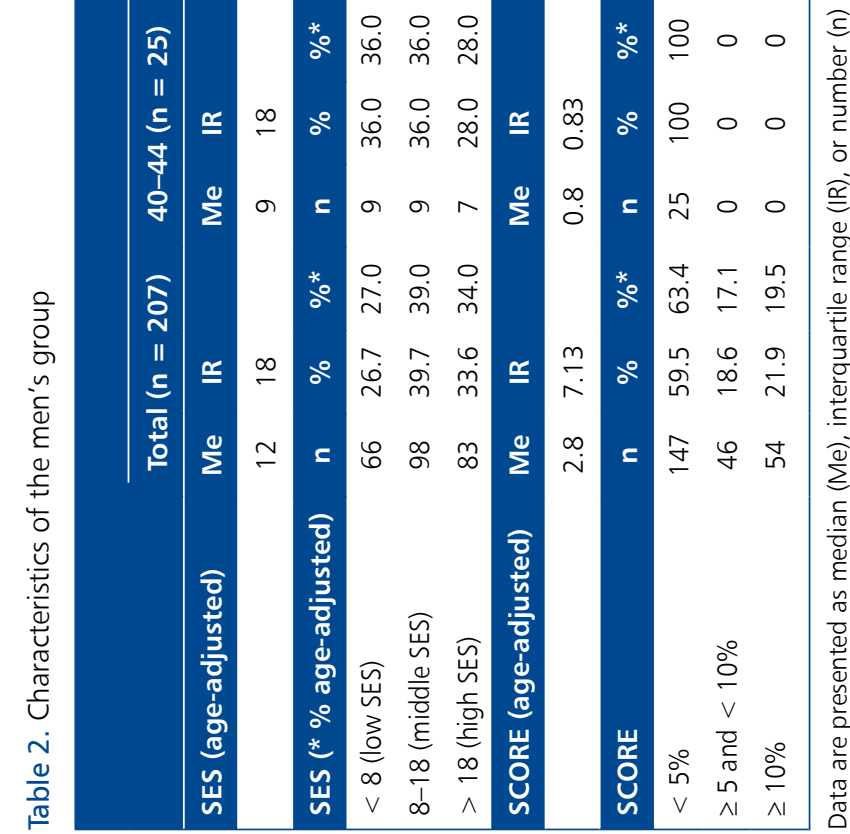


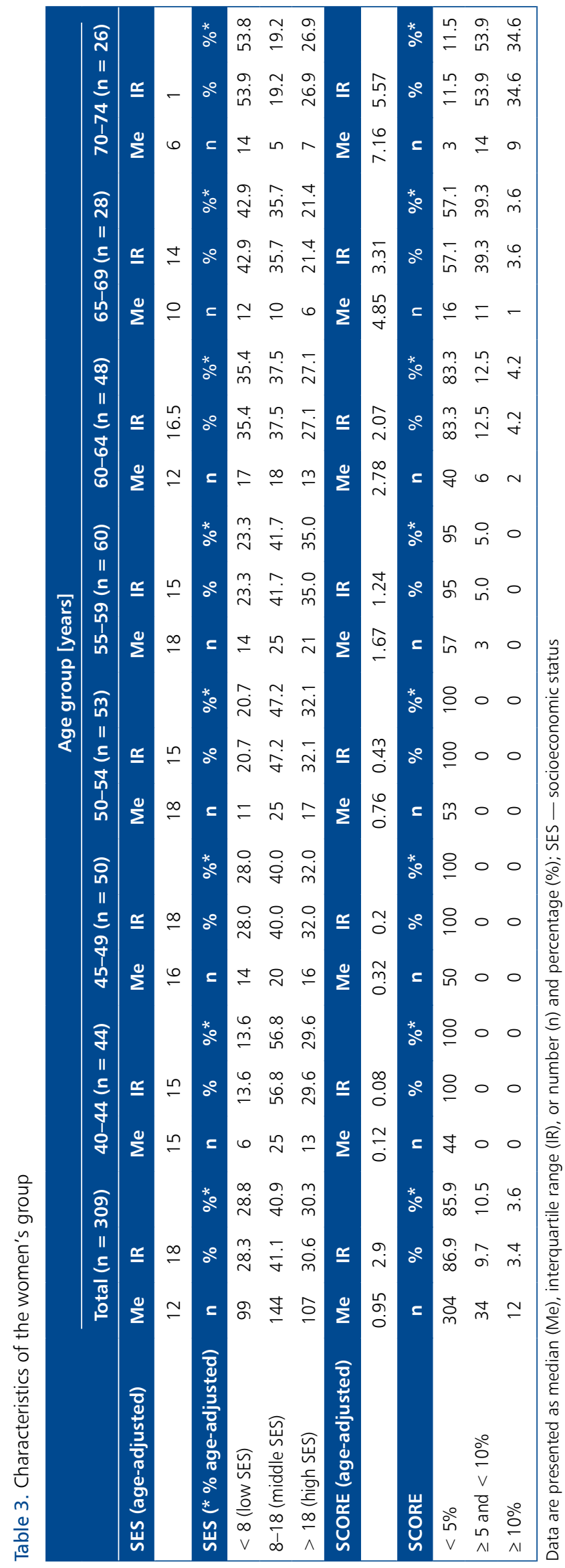

a single unit (in men it decreased by 0.076). In the analysis of individual age subgroups, distinguished from the entire group under study, we established a significant correlation between the SCORE value and SES in the age subgroups of 55 -59-year-olds $(p=0.011), 60-64$-year-olds $(p=0.014)$, and 65-69-year-olds $(p=0.034)$. A similar correlation was confirmed in the group of men aged $65-69$ years $(p=0.038)$ and women aged $40-44$ years $(p=0.003)$.

The logistic regression analysis demonstrated that, after considering the influence of age, the odds of the SCORE risk value being $\geq 10 \%$ were becoming smaller along with the increase in the SES index value in the entire group under study $(p=0.048)$ and in the men's group $(p=0.011)$. The odds ratio for the SCORE risk value being $\geq 10 \%$ depending on the SES index value was odds ratio $(\mathrm{OR})=0.978 ; 95 \%$ confidence interval $(\mathrm{Cl}) 0.956-0.999$, in the entire group, and $\mathrm{OR}=0.964 ; 95 \% \mathrm{Cl} 0.938-0.992$ in men. Furthermore, we also established that the risk of SCORE $\geq 5 \%$ decreases with the increase in the SES index value in the women's group $(p=0.042 ; \mathrm{OR}=0.970 ; 95 \% \mathrm{Cl} 0.941-0.999)$.

\section{DISCUSSION}

The correlation between SES and CVD risk was discovered as early as in 1976 by Holme et al. [12], who observed an almost doubled risk of coronary artery disease in men of low SES when compared to men of higher SES. In the analysis conducted by Tang et al. [7], a lower SES was associated with a significantly more frequent occurrence of coronary artery disease, hypertension, diabetes, and lipid disorders. In the analysis of 49 studies, Pollitt et al. [13] also confirmed the correlation between a lower SES and the increased risk of CVD. In the groups consisting of people of lower SES the risk factors of CVD such as dyslipidaemia, hypertension, insulin resistance, obesity, and tobacco smoking were more frequently observed [13].

In this study we attempted to defining the relationship between the SES and the CVD risk estimated using the SCORE scale. Based on the presented results we confirmed the correlation between low SES and increased CVD risk according to SCORE. This correlation was particularly noticeable in men aged 65-69 years and women aged 40-44 years.

The results of our study coincide with those presented by Albert et al. [14], who conducted a 10-year prospective study encompassing 22,500 women aged on average $54.1 \pm 7.1$ years to also demonstrate a relationship between lower SES and increased CVD risk. In women with lower SES, cardiovascular incidents occurred more frequently. In women with lower SES total cholesterol, LDL cholesterol, and triglyceride levels were higher when compared to those of women with higher SES. Furthermore, along with the increase in the SES, they also observed lower levels of inflammatory markers such as C-reactive protein, homocysteine, and fibrinogen [14].

In another study, which also encompassed a period of over 10 years and was conducted on the population of Fin- 
land, Lynch et al. [15] demonstrated that the highest risk of coronary artery disease, after including the typical risk factors, was present in the least educated participants, contrary to those who received better education. Increased levels of total cholesterol and LDL cholesterol, tobacco smoking, as well as lower exercise capacity and physical activity, were more often observed in participants from the lower socioeconomic group.

In our study, the correlation between high CVD risk expressed with a SCORE value and lower SES index was particularly noticeable in women aged $40-44$ years. The WOBASZ I study also confirmed a negative correlation between the SCORE risk and SES. This relation was noted in men and women aged 30-39 years, women aged 30-39 years, and women aged 40-49 years. Therefore, as presented in the study, this relationship was stronger among women, particularly from the younger age group [16]. A similar relationship was confirmed by Thurston et al. [17, 18], who conducted a multiyear research to demonstrate that the lower level of education is associated with a higher CVD risk, and this relationship is more pronounced in the women's group than in men. Women from that group had higher body mass index, lower income, more of them were diagnosed with depression, and more of them were single mothers, compared to the better educated women [17]. Kozakiewicz et al. [19] also confirmed the association between the high CVD risk and education. They observed a higher CVD risk estimated according to the SCORE algorithm in the group comprising people with lower education when compared to the better educated participants. This was related to the presence of risk factors. In women with lower education, hypertension, increased levels of LDL cholesterol and triglycerides, diabetes, and obesity were more often observed when compared to women with higher education.

The conclusion of this study is that people with lower SES face a higher risk of CVD. This correlation was particularly noticeable in men aged 65-69 years and women aged 40-44 years. This observation may have great significance because it relates to young women, who can reduce the CVD risk by means of prophylaxis. It is difficult to definitively determine what contributes to the higher risk of CVD, but it can be suspected that in families with lower SES, which typically have more members, women devote significantly less time to physical activity and they cannot afford to follow an appropriate diet. It is also difficult to assess the aspect of depression, which may more frequently apply to single mothers.

Due to the fact that the analysis used the data collected in the WOBASZ II study conducted in three voivodeships in Poland, the results cannot be regarded as representative for the entire population of Poland. In other papers regarding the results of the WOBASZ II study their authors demonstrated that CVD risk factors were more frequently present in the Lower Silesian, Silesian, and Opole voivodeships when compared to the mean value in Poland. They observed a higher percentage of overweight and obese women, especially in the Opole voivodeship [20] as well as a higher incidence of hypercholesterolaemia in both sexes in the Opole and Lower Silesian voivodeships [21]. It should be emphasised, however, that the presented issues suggest the necessity to conduct further research in this field.

\section{Limitations of the study}

The study presents the analysis of citizens in only three voivodeships. Therefore, the conclusions drawn cannot be generalised to the entire Polish population. Furthermore, the place of residence of the respondents was not considered (village or city), and the analysis was conducted only in the respective male and female age groups. Another limitation of the study is that the data collected based on the questionnaire, referring both to education and income, was obtained directly from the respondents. Therefore, it cannot be excluded that some respondents overestimated or underestimated their income, which could have affected the calculated SES value.

\section{CONCLUSIONS}

We demonstrated a statistically significant correlation between SES and CVD risk assessed according to the SCORE algorithm. The value of the CVD risk according to SCORE was inversely correlated with socioeconomic status.

\section{Acknowledgements}

The WOBASZ II project was financed from the financial resources of the Minister of Health within the framework of the health programme entitled: National Programme for the Equalisation of Accessibility to Cardiovascular Disease Prevention and Treatment for 2010-2012 POL-KARD — task: Analyses and epidemiology - "Monitoring of the epidemiological situation in Poland in the field of cardiovascular diseases."

Conflict of interest: Andrzej Pająk - honorarium from AMGEN and SANOFI not related with the present work.

\section{References}

1. Stamler J, Vaccaro O, Neaton JD, et al. Diabetes, other risk factors, and 12-yr cardiovascular mortality for men screened in the Multiple Risk Factor Intervention Trial. Diabetes Care. 1993; 16(2): 434-444, doi: 10.2337/diacare.16.2.434, indexed in Pubmed: 8432214.

2. Podolec P, Jankowski P, Zdrojewski T, et al. Polish Forum for Prevention Guidelines on Cardiovascular Risk Assessment: update 2016. Kardiol Pol. 2017; 75(1): 84-86, doi: 10.5603/KP.2017.0009, indexed in Pubmed: 28124785.

3. Mackenbach JP, Cavelaars AE, Kunst AE, et al. Socioeconomic inequalities in cardiovascular disease mortality; an international study. Eur Heart J. 2000; 21(14): 1141-1151, doi: 10.1053/euhj.1999.1990, indexed in Pubmed: 10924297.

4. Rywik SL, Piotrowski W, Broda G. [Are fluctuations in health status related to socioeconomic factors among the Polish population?]. Pol Arch Med Wewn. 2003; 109(4): 383-394, indexed in Pubmed: 12931490.

5. Brennan-Olsen SL, Williams LJ, Holloway KL, et al. Small area-level socioeconomic status and all-cause mortality within 
10 years in a population-based cohort of women: Data from the Geelong Osteoporosis Study. Prev Med Rep. 2015; 2 : 505-511, doi: 10.1016/j.pmedr.2015.05.011, indexed in Pubmed: 26844110.

6. Kaplan GA, Keil JE. Socioeconomic factors and cardiovascular disease: a review of the literature. Circulation. 1993; 88(4 Pt 1): 1973-1998, doi:10.1161/01.cir.88.4.1973, indexed in Pubmed: 8403348.

7. Tang KL, Rashid R, Godley J, et al. Association between subjective social status and cardiovascular disease and cardiovascular risk factors: a systematic review and meta-analysis. BMJ Open. 2016; 6(3): e010137, doi: 10.1136/bmjopen-2015-010137, indexed in Pubmed: 26993622.

8. Drygas W, Niklas AA, Piwońska A, et al. Multi-centre National Population Health Examination Survey (WOBASZ II study): assumptions, methods, and implementation. Kardiol Pol. 2016; 74(7): 681-690, doi: 10.5603/KP.a2015.0235, indexed in Pubmed: 26620680 .

9. Panagiotakos DB, Pitsavos CE, Chrysohoou CA, et al. The association between educational status and risk factors related to cardiovascular disease in healthy individuals: The ATTICA study. Ann Epidemiol. 2004; 14(3): 188-194, doi: 10.1016/S10472797(03)00117-0, indexed in Pubmed: 15036222.

10. Conroy RM, Pyörälä K, Fitzgerald AP, et al. SCORE project group. Estimation of ten-year risk of fatal cardiovascular disease in Europe: the SCORE project. Eur Heart J. 2003; 24(11): 987-1003, doi: 10.1016/s0195-668x(03)00114-3, indexed in Pubmed: 12788299.

11. Zdrojewski T, Jankowski P, Bandosz P, et al. [A new version of cardiovascular risk assessment system and risk charts calibrated for Polish population]. Kardiol Pol. 2015; 73(10): 958-961, doi: 10.5603/KP.2015.0182, indexed in Pubmed: 26521843.

12. Holme I, Helgeland A, Hjermann I, et al. Coronary risk factors and socioeconomic status. The Oslo study. Lancet. 1976; 2(8000): 1396-1398, indexed in Pubmed: 63860.

13. Pollitt RA, Rose KM, Kaufman JS. Evaluating the evidence for models of life course socioeconomic factors and cardiovascular outcomes: a systematic review. BMC Public Health. 2005; 5: 7 , doi: 10.1186/1471-2458-5-7, indexed in Pubmed: 15661071.
14. Albert MA, Glynn RJ, Buring J, et al. Impact of traditional and novel risk factors on the relationship between socioeconomic status and incident cardiovascular events. Circulation. 2006; 114(24): 2619-2626, doi: 10.1161/CIRCULATIONAHA.106.660043, indexed in Pubmed: 17116764.

15. Lynch J, Davey Smith G, Harper S, et al. Explaining the social gradient in coronary heart disease: comparing relative and absolute risk approaches. J Epidemiol Community Health. 2006; 60(5): 436-441, doi: 10.1136/jech.2005.041350, indexed in Pubmed: 16614335.

16. Kozakiewicz K, Podolecka E, Kwaśniewska M, et al. Association between socioeconomic status and cardiovascular risk. Kardiol Pol. 2016; 74(2): 179-184, doi: 10.5603/KP.a2015.0139, indexed in Pubmed: 26202533.

17. Thurston RC, Kubzansky LD, Kawachi I, et al. Is the association between socioeconomic position and coronary heart disease stronger in women than in men? Am J Epidemiol. 2005; 162(1): 57-65, doi: 10.1093/aje/kwi159, indexed in Pubmed: 15961587.

18. Thurston RC, El Khoudary SR, Derby CA, et al. Low socioeconomic status over 12 years and subclinical cardiovascular disease: the study of women's health across the nation. Stroke. 2014; 45(4): 954-960, doi: 10.1161/STROKEAHA.113.004162, indexed in Pubmed: 24578209.

19. Kozakiewicz K, Podolecka E, Syzdół M, et al. Relationship between education and cardiovascular risk score. Pol Arch Med Wewn. 2016; 126(5): 369-371, doi: 10.20452/pamw.3414, indexed in Pubmed: 27230774.

20. Stepaniak U, Micek A, Waśkiewicz A, et al. Prevalence of general and abdominal obesity and overweight among adults in Poland. Results of the WOBASZ II study (2013-2014) and comparison with the WOBASZ study (2003-2005). Pol Arch Med Wewn. 2016; 126(9): 662-671, doi:10.20452/pamw.3499, indexed in Pubmed: 27535012.

21. Pająk A, Szafraniec K, Polak M, et al. WOBASZ Investigators. Changes in the prevalence, treatment, and control of hypercholesterolemia and other dyslipidemias over 10 years in Poland: the WOBASZ study. Pol Arch Med Wewn. 2016; 126(9): 642-652, doi: 10.20452/pamw.3464, indexed in Pubmed:27452484.

Cite this article as: Podolecka E, Doryńska A, Nadrowski P, et al. Socioeconomic status and cardiovascular risk SCORE. Kardiol Pol. 2018; 76(3): 560-565, doi: 10.5603/KP.a2017.0253. 